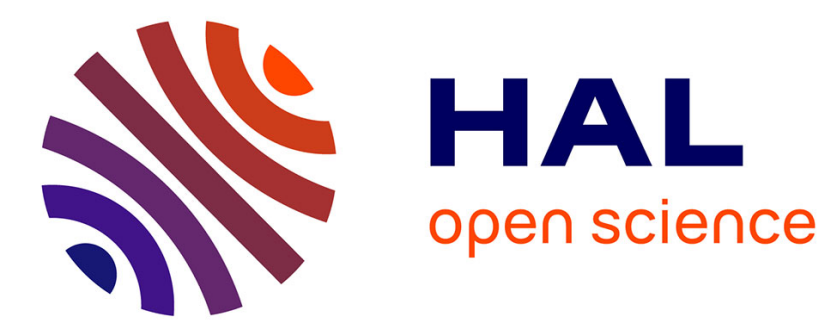

\title{
Identifying Users with Atypical Preferences to Anticipate Inaccurate Recommendations
}

\author{
Benjamin Gras, Armelle Brun, Anne Boyer
}

\section{To cite this version:}

Benjamin Gras, Armelle Brun, Anne Boyer. Identifying Users with Atypical Preferences to Anticipate Inaccurate Recommendations. Webist 2015 - 11th International Conference on Web Information Systems and Technologies, May 2015, Lisbonne, Portugal. 10.5220/0005412703810389 . hal-01254172

\section{HAL Id: hal-01254172 \\ https://hal.inria.fr/hal-01254172}

Submitted on 11 Jan 2016

HAL is a multi-disciplinary open access archive for the deposit and dissemination of scientific research documents, whether they are published or not. The documents may come from teaching and research institutions in France or abroad, or from public or private research centers.
L'archive ouverte pluridisciplinaire HAL, est destinée au dépôt et à la diffusion de documents scientifiques de niveau recherche, publiés ou non, émanant des établissements d'enseignement et de recherche français ou étrangers, des laboratoires publics ou privés. 


\title{
Identifying Users with Atypical Preferences to Anticipate Inaccurate Recommendations
}

\author{
Benjamin Gras, Armelle Brun and Anne Boyer \\ Université de Lorraine, LORIA - Campus Scientifique - 54506 Vandouvre lès Nancy \\ \{benjamin.gras, armelle.brun, anne.boyer\}@loria.fr
}

\begin{abstract}
Keywords: Atypical preferences, Atypical users, Recommender systems, Collaborative filtering, Accuracy of recommendations

Abstract: The social approach in recommender systems relies on the hypothesis that users' preferences are coherent between users. To recommend a user some items, it uses the preferences of other users, who have preferences similar to those of this user. Although this approach has shown to produce on average high quality recommendations, which makes it the most commonly used approach, some users are not satisfied. Being able to anticipate if a recommender will provide a given user with inaccurate recommendations, would be a major advantage. Nevertheless, little attention has been paid in the literature to studying this particular point. In this work, we assume that a part of the users who are not satisfied do not respect the assumption made by the social approach of recommendation: their preferences are not coherent with those of others; they have atypical preferences. We propose measures to identify these users, upstream of the recommendation process, based on their profile only (their preferences). The experiments conducted on a state of the art corpus show that these measures allow to identify reliably a subset of users with atypical preferences, who will get inaccurate recommendations.
\end{abstract}

\section{INTRODUCTION}

The continuous increase of the amount of data available on the Internet makes the task of accessing targeted information more and more complex for users. This is the reason why many services now offer to assist users during their search, by selecting for them the most relevant information or data. Several types of such services are proposed, among which recommender systems (RS) (Goldberg et al., 1992). Through a recommendation process, a RS aims to guide users towards resources relevant for them. A resource can be a book, a movie, a web page, etc. To make such a recommendation possible, the system uses the knowledge it has collected about the active user, (the user the system aims to provide with recommendations).

RS have been studied for more than twenty years (Goldberg et al., 1992). The two most common approaches are content-based filtering (Bobadilla et al., 2013) and collaborative filtering (CF) (Resnick et al., 1994; Su and Khoshgoftaar, 2009). Content-based filtering exploits the content of the resources (as well as index, keywords, title, type of the resource, etc.) to select those that match the active user's preferences.
Conversely, CF (also referred to as social filtering) does not require the exploitation of the content of the resources. It relies on the assumption that users' preferences are consistent among users, which allows to infer the active user's preferences from those of other users. In both approaches, users' preferences are generally represented by ratings on resources. As CF is the most popular approach, it will be the focus of this work.

Providing users with high quality recommendations is of the highest importance. In the context of e-commerce it increases customer retention, in elearning it improves learners' learning process, etc. The quality of the recommendations provided by $\mathrm{CF}$ is on average considered as acceptable (Castagnos et al., 2013). However, some users do not receive accurate recommendations, which results in serious consequences: unsatisfied users, user attrition, failure among learners, etc.

We think it is essential that a recommender can anticipate, upstream of the recommandation process, which users it will provide with inaccurate recommendations. Once these users are identified, the system can decide to not provide them with recommendations, or use another approach. The literature has 
shown that one reason why some users are not satisfied is the small number of preferences the system collected about them. This problem is referred to as the cold start problem (Schein et al., 2001). However, some users with a significant number of preferences still get inaccurate recommendations. Inaccurate recommendations can also be explained by the quality of the preferences collected about these users (Grcar et al., 2005) or by the inconsistency of users when expressing their preferences (Ekstrand, 2014). Recent works have noticed that some users tend to rate resources differently than others (Del Prete and Capra, 2010). Remind that collaborative filtering assumes that preferences (ratings) are consistent between users. As these users do not match this requirement (their preferences are different from those of others), this may explain why some of them get inaccurate recommendations. We will refer their preferences to as atypical preferences.

The work conducted in this paper is in line with these latter works. We aim at identifying reliably users with atypical preferences (ratings) and who will receive inaccurate recommendations. From now on, we will refer these users to as atypical users. Their identification will be performed prior to any recommendation computation. To reach these goals, we propose several measures that reflect the atypicity of preferences of a user.

Section 2 presents a short overview of recommender systems and the way atypical users are identified and managed in social recommendation. Section 3 introduces three measures we propose to identify atypical users. Then, in Section 4 the experiments we conducted to evaluate those measures are presented. Finally, we conclude and discuss our work in the last section.

\section{RELATED WORKS}

\subsection{Social Recommender Systems}

Social recommendation, also denoted by collaborative filtering $(\mathrm{CF})$, relies on the knowledge of users' preferences (generally some ratings) on some resources to provide a user, referred to as the active user, with some personalized recommendations. One way to perform such recommendations is by inferring the active user's unknown preferences, then recommending the most relevant resources for him/her. When ratings are not available, they can be inferred from the traces of activity left by the users (Ormándi et al., 2010).
There are two main approaches in social recommendation: the memory-based approach and the model-based approach (Adomavicius and Tuzhilin, 2005). The memory-based approach exploits directly users' preferences, without pre-processing. The most commonly used technique, the $K N N$ ( $K$ Nearest Neighbors), computes the similarities of preferences between the active user and each other user, then selects the $K$ nearest users to the active user. This approach is simple to implement, provides high quality recommendations and takes into account each new preference dynamically in the recommendation process. However, it does not scale, due to the computation cost of the similarities. The model-based approach learns, as its name suggests, a model that describes the data (preferences), i.e. the relationship between users and resources. This model is used to estimate unknown preferences and to provide the active user with recommendations. This approach does not suffer so much from the scalability problem. However, it does not easily allow dynamic changes in the model, especially if it has to be updated each time a new preference is provided by a user. The modelbased matrix factorization technique has emerged few years ago and is now the most commonly used technique, due to the quality of recommendations it provides. The matrix of users' preferences is factorized into two sub-matrices, one representing users, the other representing the resources, both in a common sub-space where dimensions correspond to latent features. There are several techniques of matrix factorization, including the singular value decomposition (Billsus and Pazzani, 1998) and alternating least squares (Hu et al., 2008).

\subsection{Identifying Atypical Users in Recommender Systems}

In the literature, several terms are used to make reference to atypical users. They are deviant users, abnormal users, grey sheeps, etc. (Del Prete and Capra, 2010; Ghazanfar and Prugel-Bennett, 2011). Most of the techniques used to perform their identification are issued from data analysis. The abnormality measure (Del Prete and Capra, 2010; Haydar et al., 2012) is the most commonly used one. It has actually several names: abnormality, deviance coefficient, deviance, etc. but they all reflect the same phenomenon, namely the tendency of a user to rate differently from others. This measure uses the difference between the ratings assigned by a user on some resources and the average rating on these resources. It is defined by equation (1). 


$$
\operatorname{Abnormality}(u)=\frac{\sum_{r \in R_{u}}\left|n_{u, r}-\overline{n_{r}}\right|}{\left\|R_{u}\right\|}
$$

where $n_{u, r}$ represents the rating that user $u$ assigned to resource $r, \overline{n_{r}}$ is the average rating of $r, R_{u}$ is the set of resources rated by $u$ and $\left\|R_{u}\right\|$ is their number. Users with a high abnormality are considered as atypical users. The main advantage of this measure is its low complexity. However, although this measure is the reference measure in the literature to identify users with atypical preferences, from our point of view it suffers from several limitations. First, the resources about which users' preferences are not unanimous (the ratings between users is very different) will unfairly increase the abnormality of the users who rate these resources. Second, this measure does not take into account the individual behavior of each user. For example, a user more strict than the average user may be labelled as abnormal, while he/she has similar preferences to others, he/she only differs in his/her way of rating resources. This measure will thus probably identify some users as atypical, whereas they will get accurate recommendations.

Some studies identify atypical users with the aim to explain the fluctuations of performance of RS (Bellogín et al., 2011; Haydar et al., 2012; Griffith et al., 2012; Ekstrand and Riedl, 2012), by studying users' characteristics (number of ratings, number of neighbors, etc.). For example, a link between the small number of ratings of a user and a high recommendation error may be identified (cold start problem). In (Haydar et al., 2012), the authors form clusters of users, based on their preferences and aim at interpreting the clusters. Among the set of clusters, a cluster made up of atypical users is identified: users with a high recommendation error (RMSE) and who have a high abnormality (equation (1)) as well. However, we are convinced that in the general case, clustering fails to build a cluster of users with atypical and who will get inaccurate recommendations. Indeed, an atypical user, in the sense of the social recommendation, has preferences that are not close to those of other users. Thus, if a user belongs to a cluster, it means that his/her preferences are similar to those of users in the same cluster. So, he/she is not an atypical user. The work presented in (Ghazanfar and PrugelBennett, 2011) also relies on clustering of users, and is in line with our convinction: it proposes to consider users who are far from the center of their cluster as atypical users.

(Bellogín et al., 2011) defines a clarity indicator, that represents how much a user is non-ambiguous in his/her ratings. This indicator is based on the entropy measure: a user is considered as ambiguous (small value of clarity) if his/her ratings are not stable across resources. Authors show that there is a link between the ambiguity of the ratings of a user and the quality of recommendations he/she gets. Users with a small clarity value are considered as noise and are discarded from the system; they do not receive any recommendations. We believe that this approach quickly reaches its limits. Indeed, various ratings (preferences) of a user can be explained by several factors such as the evolution of his/her preferences through time, his/her varying preferences across domains, etc. Therefore, a social approach may anyway provide this user with high quality recommendations. Notice that, at the opposite of previous approaches, the clarity indicator does not reflect the coherence of a user's preferences with respect to other users, it reflects the coherence he/she has with him/herself. It can thus be exploited in an approach other than the social one. Clarity can also be linked the magic barrier concept (Herlocker et al., 2004) and to recent works about user inconsistency and natural variability (Bellogín et al., 2014), which aim at estimating an upper bound on the rating prediction accuracy.

The impact of users identified as atypical on the overall quality of recommendations has been studied. The comparison of the results presented is difficult as atypical users are not selected on the basis of the same criteria. However, they do all conclude that removing atypical users in the learning phase of the recommender improves the overall quality of the recommendations.

Notice that the identification of atypical users may be associated with the identification of outliers. According to (Hawkins, 1980), an outlier is "an observation that deviates so much from other observations as to arouse suspicion that is was generated by a different mechanism". In the context of recommender systems, an outlier is a user whose preferences appear to have been generated by a different preference expression mechanism. Criterion based, statistical approaches and clustering are also widely used in the field of outliers detection (Aggarwal, 2013).

\subsection{Managing Atypical Users in Recommender Systems}

Once atypical users have been identified, one question that can be addressed is related to their management. In the context of recommender systems, new recommendation approaches dedicated to these specific profiles are developed, to provide them with better recommendations. In (Del Prete and Capra, 2010), which refer atypical users to as deviant users, the authors divide users into two subsets: deviant and non- 
deviant users, using the abnormality measure (equation (1)). These two sets are considered independently when training recommendation models (two models are formed), as well as during the recommendation process. Only deviant users are taken into account when the active user is identified as deviant. Conversely, only non-deviant users are considered when the active user is non-deviant. This approach has shown to improve the quality of recommendations related to non-deviant users. However, it has no impact on the quality of the recommendations provided to deviant users. This confirms our intuitions that atypical users do not share preferences with any of the users. In addition, we find this result not surprising as the recommendation approach has not been adapted to these specific users.

We previously reported how (Ghazanfar and Prugel-Bennett, 2011) identify atypical users through clustering. To address these atypical users, they use a specific cluster based CF algorithm (model approach) to better reflect the preferences of these users and to offer them better recommendations. Authors assume that these users have only partial agreement with the rest of the community (i.e. CF will fail on these users) and propose to rely on content of resources to generate recommendations.

J. Bobadilla (Bobadilla et al., 2012) has proposed a more general solution to take into account the specificities of atypical users, through a new similarity measure. This new measure is based on the singularity of ratings. A rating on a resource is considered as singular if it does not correspond to the majority rating on this resource. Authors assume that atypical users tend to assign singular ratings to resources. When computing the similarity between two users, the more a rating is singular, the greater its importance. The similarity between users is then used as in a classical $K N N$-based recommendation approach. It has shown to provide high quality preferences to users with specific preferences.

\section{New Identification Measures of Atypical Users}

We propose in this section new measures for identifying atypical users, i.e. users with preferences that differ from those of the population of users and who will receive low quality recommendations. These identification measures are designed to be used prior to the recommendation process, so they rely on the users' profiles (preferences on resources) only.

\subsection{CorrKMax}

The first measure we propose is dedicated to the neighborhood-based recommendation approach, namely the $K N N$ technique. We are convinced that the $K N N$ approach, which exploits the $K$ most similar users to the active user, fails in the case of active users who do not have enough highly similar users. We thus define CorrKMax to highlight the link between the similarity of the most similar users of a user and the quality of the recommendations he/she gets. CorrKMax(u) (equation (2)) represents the average similarity between the active user $u$ and his/her $K$ most similar users.

$$
\operatorname{CorrKMax}(u)=\frac{\sum_{n \in \operatorname{Neigh}(u)} \operatorname{Pearson}(u, n)}{\|\operatorname{Neigh}(u)\|}
$$

where Pearson $(u, n)$ is the Pearson correlation between the preferences of users $u$ and $n$. Neigh $(u)$ represents the $k$ most similar users to $u$, in the limit their correlation with $u$ is positive. We believe that the users associated with a low value of $\operatorname{CorrKMax}(u)$ receive inaccurate recommendations.

The two following measures are an extension of the Abnormality measure from the state of the art, which has shown good atypical user identification capabilities (see section 2.2). To overcome the limitations that we have mentioned and presented in the previous section, we propose a first improvement.

\subsection{AbnormalityCR}

The AbnormalityCR (Abnormality with Controversy on Resources) measure assumes that the meaning of the discrepancy between a rating on a resource and the average rating of this resource differs according to the resource. Indeed, a large discrepancy on a controversial resource has not the same meaning as a large discrepancy on a consensual resource. The abnormality measure of the state of the art considers these differences as equal, which has the effect of increasing the abnormality of users who express their preferences on controversial resources. We therefore propose to reduce the impact of the ratings on controversial resources, by weighting them with the degree of controversy of the resources they refer to.

This degree of controversy of a resource is based on the standard deviation of the ratings on this resource. The AbnormalityCR of a user $u$ is computed as shown in equation (3).

$$
\text { Abnormality }_{C R}(u)=\frac{\sum_{r \in R_{u}}\left(\left(n_{u, r}-\overline{n_{r}}\right) * \text { contr }(r)\right)^{2}}{\left\|R_{u}\right\|}
$$


where contr $(r)$ represents the controversy associated with resource $r$. It is based on the normalized standard deviation of ratings on $r$ and is computed according to equation (4).

$$
\operatorname{contr}(r)=1-\frac{\sigma_{r}-\sigma_{\min }}{\sigma_{\max }-\sigma_{\min }}
$$

where $\sigma_{r}$ is the standard deviation of the ratings associated with the resource $r$. $\sigma_{\min }$ and $\sigma_{\max }$ are respectively the smallest and the largest possible standard deviation values, among resources. The computation complexity of AbnormalityCR is comparable to that of the abnormality of the state of the art. It can therefore be computed frequently and thus take into account new preferences.

\subsection{AbnormalityCRU}

The AbnormalityCRU (Abnormality with Controversy on Resources and Users) measure is a second improvement of Abnormality. It starts from the observation that neither Abnormality $(u)$ nor AbnormalityCR $(u)$ reflect the general behavior of the user $u$. Thus, a user who is strict in his/her way to rate resources may be considered as atypical, even if his/her preferences are actually not. In addition, this user will probably receive high quality recommendations. To avoid this bias, we propose to center the ratings of each user around his/her average rating. This way to reflect the user's behavior is also that used in the Pearson correlation coefficient. Furthermore, the average rating of a resource is computed on the centered ratings. The abnormality of a user $u$, denoted by AbnormalityCRU $(u)$, is computed using equation (5).

$$
\begin{aligned}
& \operatorname{Abnormalit}_{C R U}(u)= \\
& \qquad \frac{\sum_{r \in R_{u}}\left[\left(\left|n_{u, r}-\overline{n_{u}}-\overline{n_{C_{r}}}\right|\right) * \operatorname{contr}_{C}(r)\right]^{2}}{\left\|R_{u}\right\|}
\end{aligned}
$$

where $\overline{n_{C_{r}}}$ represents the average centered rating of resource $r$, contr $r_{C}(r)$ represents the controversy associated with resource $r$, computed from the standard deviation of the ratings on $u$, centered with respect to users. The computation of AbnormalityCRU $(u)$ is more complex than Abnormality $C R(u)$, but should allow a more accurate identification of atypical users.

Note that these last two measures are independent of the recommendation approach used, whether it is $K N N$ or matrix factorization, contrary to the CorrKMax measure.

\section{Experiments}

The experiments we conduct in this section are intended to assess the quality of the atypical users identification measures we propose, in comparison with the measures from the state of the art. The assessment is based on the quality of the recommendations provided to users identified as atypical.

\subsection{Dataset \& System Settings}

Experiments are conducted on the MovieLens100K dataset from the state of the art. MovieLens $100 \mathrm{~K}$ is made up of 100,000 ratings from 943 users on 1,682 movies (resources). The ratings range from 1 to 5 , on integer values. We divide the dataset into two sub-sets made up of $80 \%$ (for learning) and $20 \%$ (for test) of the ratings of each user.

As presented in the beginning of this paper, we aim at identifying users who will be provided with inaccurate recommendations, due to their atypical preferences. The literature emphasizes that users about who the system has collected few preferences get inaccurate recommendations (cold start problem). To not bias our evaluation, we decide to discard these users from the dataset. We consider that a user who has less than 20 ratings in the training set is associated to cold start (Schickel-Zuber and Faltings, 2006). The set of users is then reduced to 821 users.

The accuracy of recommendations is evaluated through standard measures, which compute the discrepancy between the rating provided by a user on a resource and the rating estimated by the recommender. These measures are the Mean Absolute Error (MAE) and the Root Mean Square Error (RMSE). The lower MAE and RMSE, the higher the accuracy of recommendations provided to users. In this work, we will specifically exploit per-user MAE $(M A E(u))$ and RMSE $(R M S E(u))$, computed by equations (6) and (7).

$$
\begin{gathered}
\operatorname{MAE}(u)=\frac{\sum_{r \in R_{u}}\left|n_{u, r}-n_{u, r}^{*}\right|}{\left\|R_{u}\right\|} \\
\operatorname{RMSE}(u)=\sqrt{\frac{\sum_{r \in R_{u}}\left(n_{u, r}-n_{u, r}^{*}\right)^{2}}{\left\|R_{u}\right\|}}
\end{gathered}
$$

where $n_{u, r}^{*}$ is the estimated rating of user $u$ on resource $r$.

To compute the per user errors, we implement two different techniques of CF: a User-Based recommender and a Matrix Factorization technique for recommendation. In this way, we want to show the genericity of our measures. We set up the most used 
settings in the state of the art for each of these two approaches.

The User-Based recommender define the similarity of two users as the Pearson correlation coefficient of their two ratings vectors. The rating estimation for a user is based on the ratings of his $\mathrm{k}$ nearest neighbors, using a weighted average of their ratings. We fix $\mathrm{k}=$ 20 for this dataset.

The matrix factorization technique is a model based approach decomposing the User-Item rates matrix into two sub matrices, one representing users and the other representing items. Each user or item is represented by $\mathrm{n}$ latent features. We use the ALS factorization technique to compute this decomposition with 5 latent features. We use then this model to calculate rate estimations.

We then focus on the correlation between errors calculated with those approaches and our identification measures.

\subsection{Correlations Between Identification Measures and Recommendation Error}

In this section, we evaluate the quality of the atypical users identification measures, through their correlation with the recommendation error (per-user MAE and per-user RMSE) of the $K N N$ technique (with $K=20$ ). Four measures are studied: Abnormality from the state of the art and the three measures we propose: AbnormalityCR and AbnormalityCRU and CorrKMax (with $K=20$ also). Based on these correlations, we can determine which measures are good indicators of the quality of recommendations that will be proposed to users. The correlations are presented in Table 1.

\begin{tabular}{|l||c|c|}
\hline & MAE & RMSE \\
\hline \hline Abnormality & 0.441 & 0.453 \\
\hline Abnormality $_{C R}$ & $\mathbf{0 . 5 0 3}$ & $\mathbf{0 . 5 0 4}$ \\
\hline Abnormality $_{C R U}$ & $\mathbf{0 . 5 3 8}$ & $\mathbf{0 . 5 4 6}$ \\
\hline CorrKMax $^{\prime}$ & -0.17 & -0.22 \\
\hline
\end{tabular}

Table 1: Correlations between identification measures and MAE/ RMSE of a $K N N$ approach

The Abnormality measure from the state of the art has a correlation of 0.441 and 0.453 with MAE and RMSE respectively. These correlations are significant and confirm the existence of a link between the Abnormality of a user and the accuracy of the recommendation he/she gets: the higher the Abnormality of a user, the lower the accuracy of the recommendations he/she receives. At the opposite, the lower the Abnormality, the higher the accuracy. Recall that a user with a high Abnormality value is considered as atypical.

When using AbnormalityCR, the correlation with RMSE reaches 0.504 , which corresponds to an improvement of $11 \%$ of the correlation. We can deduce that integrating the controversy associated with the resources in the computation of the $\mathrm{Ab}$ normality improves the estimation of the accuracy of the recommendations provided to users. With AbnormalityCRU, the correlation is equal to 0.546 , which corresponds to a further improvement of $8 \%$ (20\% with respect to Abnormality). So, taking into account users' rating peculiarities (users' profile) further improves the anticipation of the accuracy of recommendations.

The low correlation between CorrKMax and RMSE (-0.22) indicates that, contrary to our intuition, the quality of a user's neighborhood is not correlated with the quality of the recommendations provided to him/her, with a $K N N$ recommendation technique. This result is surprising as the $K N N$ technique assumes that the more a user is correlated with the active user, the more he/she is reliable, and thus the more important he/she is in the computation of recommendations for the active user.

We also notice here that all identification measures are more correlated with the RMSE than with the MAE. We therefore choose to use the RMSE as the error measure in the following experiments.

\subsection{Recommendation Error for Atypical Users}

A correlation is used to evaluate the relationship between two variables on a set of observations. However, there may be a relationship within only a subset of the observations of these variables. In that case, the correlation may not allow to identify this relationship. In particular, in this paper we aim at identifying a link between atypical users and the RMSE. Therefore, in the following experiments, we will no more focus on the correlation between identification measures and RMSE, but on the distribution of the errors observed on users identified as atypical. The users with an extreme value of the identification measure are considered as atypical (the highest ones for the abnormality measures).

To study the distribution of these errors, we extract the minimum, the maximum, the quartiles and the median of the errors (RMSE), and depict them with box plots. The four identification measures: Abnormality, AbnormalityCR, AbnormalityCRU and CorrKMax are studied.

To evaluate precisely these four measures, we 


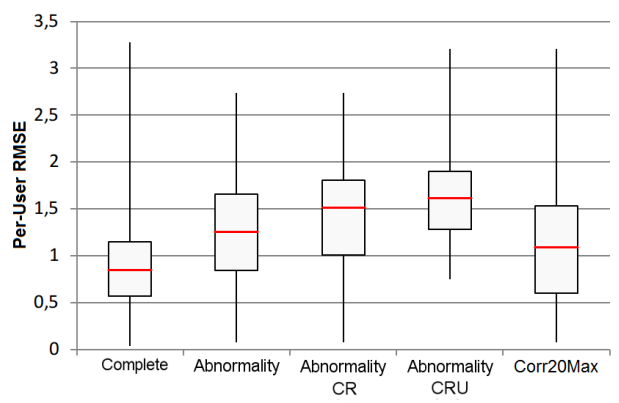

Figure 1: Distribution of RMSE of atypical users with the KNN technique

compare their box plots with the one of the complete set of users (denoted by Complete in Figures 1 and 2). The higher the errors, the more accurate the measure. As the identification measures do not all have comparable values, we did not use a predefined atypicity threshold value. We chose to consider a predetermined percentage of atypical users, which we fixed experimentally at $6 \%$ of the complete set of users. This corresponds to about 50 users among the 821 users. We compare these measures in the framework of the two main recommendation techniques: the $K N N$ technique, and the matrix factorization technique.

\subsubsection{Errors Associated with Atypical Users in the KNN Technique}

The distribution of the errors obtained with the $K N N$ technique, according to the identification measure, are presented in Figure 1.

The median error on the complete set of users (Complete) is 0.82 . The median error associated with Abnormality is 1.26. This represents an increase in the error by over $50 \%$. Furthermore, the median value of Abnormality is equivalent to the third quartile of the Complete set: $50 \%$ of users identified as atypical users with Abnormality are part of the $25 \%$ of users with the highest RMSE in the Complete set. However, $25 \%$ of the users considered as atypical have a RMSE lower than the median RMSE of the complete set of users. This means that, although Abnormality from the state of the art allows to identify users who will receive inaccurate recommendations, it appears to select a significant number of users who will receive accurate recommendations (false detection). Abnormality is thus not precise enough. Recall that users identified as atypical may either not receive any recommendations at all, or may get recommendations from another approach, which may be less accurate. The precision of the measure used is thus of the highest importance. The limits that we

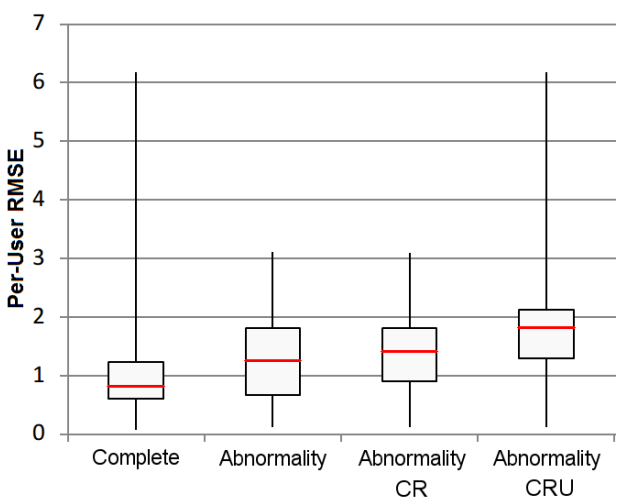

Figure 2: Distribution of RMSE of atypical users with the matrix factorization technique

presented in the previous section are confirmed: the discrepancy between a rating and the average rating on a resource is not sufficient to reliably predict inaccurate recommendations.

Both measures AbnormalityCR and AbnormalityCRU are of higher quality than Abnormality. AbnormalityCRU appears to be the best one. With AbnormalityCRU, all the users identified as atypical users have a RMSE higher than the median RMSE of the complete set of users. In addition, over $75 \%$ of these users have a RMSE higher than 1.25 , i.e. $75 \%$ of the users with the highest AbnormalityCRU are among the $25 \%$ of the complete set of users who will receive inaccurate recommendations. The precision of the AbnormalityCRU measure is thus high.

However, once more CorrKMax $(K=20)$ is not precise, the users identified as atypical tend to receive high quality recommendations (50\% of them). The low similarity of a user's nearest neighbors is thus not a reliable information to predict the quality of recommendations this user will receive.

We can conclude that, when the AbnormalityCRU measure identifies a user as an atypical user, he/she will actually receive inaccurate recommendations with the $K N N$ recommendation technique.

\subsubsection{Errors Associated with Atypical Users in the Matrix Factorization Technique}

In this section, we seek to assess whether the identification measures studied are generic, i.e. if they can also be used with a matrix factorization technique. The errors associated with CorrKMax are not studied here, as CorrKMax is dedicated to the KNN technique. Figure 2 presents the distributions of the errors of the three Abnormality measures with a matrix factorizaton approach. 
The ranking of the measures obtained on the $K N N$ technique is confirmed here: both Abnormality $C R$ and AbnormalityCRU improve Abnormality; AbnormalityCRU remains the most accurate measure for identifying atypical users. Moreover, we can observe that the quality of AbnormalityCRU is similar to the one observed with the $K N N$ technique: $75 \%$ of users identified as atypical receive recommendations of quality equivalent to the worse $25 \%$ of recommendations from the complete set of users.

In conclusion, we can say that the AbnormalityCRU measure, which we propose, is the most accurate measure: when it identifies a user as atypical, he/she most likely will receive low quality recommendations. Moreover, this measure is independent of the recommendation technique: it is efficient in both $K N N$ and matrix factorization techniques. This may be linked with the conclusion made in (Ekstrand, 2014), which has shown that different recommendation approaches (collaborative user-user, collaborative item-item, content, etc.) tend to fail on the same users.

However, although the high accuracy of AbnormalityCRU, some users (from the complete set) with a high RMSE are identified by none of the Abnormality measures: it concerns $50 \%$ of the users who have a RMSE greater than 2. This means that further work has to be conducted to identify the characteristics of these users.

\section{Conclusion and Perspectives}

Social recommender systems is the context of this work. Our objective was to identify users who will receive inaccurate recommendations, upstream of the recommendation process, i.e. based only on the characteristics of their preferences. We hypothesized that users with preferences that differ from those of other users will receive inaccurate recommendations. We have referred these users to as atypical users. To validate this hypothesis, we proposed several measures for identifying atypical users, based on the similarity of users' preferences with other users, on the average discrepancy of the ratings they provide in comparison with the average rating of other users, on the consensus of ratings on resources, or on users rating profile. We have shown, on a state of the art dataset, that the measure that uses all these criteria is the most accurate one and allows to reliably anticipate that a user will get inaccurate recommendations, with either a $K N N$ or a matrix factorisation technique.

In a further work, we will focus on the proposition of a new recommendation approach, to provide atyp- ical users with high quality recommendations. In parallel, it will be interesting to investigate the reasons why some users do get inaccurate recommendations and are not identified by any of the measures studied, as mentionned in the previous section. Specifically, a user may be atypical on a subset of items, which is not considered by the measures studied here.

\section{REFERENCES}

Adomavicius, G. and Tuzhilin, A. (2005). Toward the next generation of recommender systems: A survey of the state-of-the-art. IEEE transactions on knowledge and data engineering, 17(6):734-749.

Aggarwal, C. (2013). An introduction to outlier analysis. In Outlier Analysis, pages 1-40. Springer New York.

Bellogín, A., Castells, P., and Cantador, I. (2011). Predicting the performance of recommender systems: An information theoretic approach. In Proc. of the Third Int. Conf. on Advances in Information Retrieval Theory, ICTIR'11, pages 27-39. Springer.

Bellogín, A., Said, A., and de Vries, A. (2014). The magic barrier of recommender systems no magic, just ratings. In Proc. of the 22nd Conf. on User Modelling, Adaptation and Personalization (UMAP).

Billsus, D. and Pazzani, M. J. (1998). Learning collaborative information filters. In Proc. of the Fifteenth Int. Conf. on Machine Learning, ICML '98, pages 46-54, San Francisco, CA, USA. Morgan Kaufmann Publishers Inc.

Bobadilla, J., Ortega, F., and Hernando, A. (2012). A collaborative filtering similarity measure based on singularities. Inf. Process. Manage., 48(2):204-217.

Bobadilla, J., Ortega, F., Hernando, A., and Gutiérrez, A. (2013). Recommender systems survey. Know.-Based Syst., 46:109-132.

Castagnos, S., Brun, A., and Boyer, A. (2013). When diversity is needed... but not expected! In IMMM 2013, The Third Int. Conf. on Advances in Information Mining and Management.

Del Prete, L. and Capra, L. (2010). differs: A mobile recommender service. In Proc. of the 2010 Eleventh Int. Conf. on Mobile Data Management, MDM '10, pages 21-26, Washington, USA. IEEE Computer Society.

Ekstrand, M. (2014). Towards Recommender Engineering. Tools and Exp. for Identifying Recommender Differences. PhD thesis, Faculty of the University of Minnesota.

Ekstrand, M. and Riedl, J. (2012). When recommenders fail: predicting recommender failure for algorithm selection and combination. In Proc. of the sixth ACM conf. on recommender systems, pages 233-236. ACM.

Ghazanfar, M. and Prugel-Bennett, A. (2011). "fulfilling the needs of gray-sheep users in recommender systems, a clustering solution". In 2011 Int. Conf. on Information Systems and Computational Intelligence. Event Dates: 18-20, January. 
Goldberg, D., Nichols, D., Oki, B., and Terry, D. (1992). Using collaborative filtering to weave an information tapestry. Communications of the ACM, 35(12):61-70.

Grcar, M., Mladenic, D., and Grobelnik, M. (2005). Data quality issues in collaborative filtering. In Proc. of ESWC-2005 Workshop on End User Aspects of the Semantic Web.

Griffith, J., O'Riordan, C., and Sorensen, H. (2012). Investigations into user rating information and predictive accuracy in a collaborative filtering domain. In Proc. of the 27th Annual ACM Symposium on Applied Computing, SAC '12, pages 937-942, New York. ACM.

Hawkins, D. M. (1980). Identification of outliers, volume 11. Springer.

Haydar, C., Roussanaly, A., and Boyer, A. (2012). Clustering users to explain recommender systems' performance fluctuation. In Foundations of Intelligent Systems, volume 7661 of Lecture Notes in Computer Science, pages 357-366. Springer.

Herlocker, J., Konstan, J., Terveen, L., and Riedl, J. (2004). Evaluating collaborative filtering recommender systems. ACM Transactions on Information Systems.

Hu, Y., Koren, Y., and Volinsky, C. (2008). Collaborative filtering for implicit feedback datasets. In Proc. of the 2008 Eighth IEEE Int. Conf.e on Data Mining, ICDM '08, pages 263-272, Washington, DC, USA. IEEE Computer Society.

Ormándi, R., Hegeds, I., Csernai, K., and Jelasity, M. (2010). Towards inferring ratings from user behavior in bittorrent communities. In Proc. of the 2010 19th IEEE Int. Workshops on Enabling Technologies: Infrastructures for Collaborative Enterprises (WETICE), pages 217-222.

Resnick, P., Iacovou, N., Suchak, M., Bergstrom, P., and Riedl, J. (1994). Grouplens: An open architecture for collaborative filtering of netnews. In Proc. of the 1994 ACM Conf. on Computer Supported Cooperative Work, CSCW'94, pages 175-186, New York. ACM.

Schein, A. I., Popescul, A., Ungar, L. H., and Pennock, D. (2001). Generative models for cold-start recommendations. In Proc. of the 2001 SIGIR workshop on recommender systems.

Schickel-Zuber, V. and Faltings, B. (2006). Overcoming incomplete user models in recommendation systems via an ontology. In Proc. of the 7th Int. Conf. on Knowledge Discovery on the Web, WebKDD'05, pages 3957, Berlin. Springer.

Su, X. and Khoshgoftaar, T. M. (2009). A survey of collaborative filtering techniques. Adv. in Artif. Intell., 2009:4:2-4:2. 